\title{
Preoperative Cytologic Diagnosis of Noninvasive Follicular Thyroid Neoplasm with Papillary-Like Nuclear Features: A Prospective Analysis
}

\author{
Kyle C. Strickland, Marina Vivero,, Vickie Y. Jo,, Alarice C. Lowe, Monica Hollowell,, Xiaohua Qian, \\ Tad J. Wieczorek, ${ }^{1}$ Christopher A. French,, Lisa A. Teot,, Peter M. Sadow, Erik K. Alexander, \\ Edmund S. Cibas, Justine A. Barletta, ${ }^{1, *}$ and Jeffrey F. Krane ${ }^{1, *}$
}

Background: The term noninvasive follicular thyroid neoplasm with papillary-like nuclear features (NIFTP) has been proposed to replace noninvasive follicular variant of papillary thyroid carcinoma (FVPTC) in recognition of the indolent behavior of this tumor. The ability to differentiate NIFTP from classical papillary thyroid carcinoma (cPTC) by fine-needle aspiration (FNA) would facilitate conservative management for NIFTP. The aim of this study was to determine if NIFTP can be distinguished prospectively from cPTC.

Methods: From June 2015 to January 2016, thyroid FNAs with a diagnosis of "malignant" or "suspicious for malignancy" were prospectively scored for features associated with NIFTP/FVPTC (microfollicular architecture) or cPTC (papillae, psammomatous calcifications, sheet-like architecture, and nuclear pseudoinclusions) and categorized as NIFTP/FVPTC, cPTC, or indeterminate. Results were correlated with subsequent histologic diagnoses. Results: The study included 52 patients with 56 resected nodules with a cytologic diagnosis of "malignant" (43/56) or "suspicious for malignancy" (13/56). Forty-nine patients (94\%) underwent initial total thyroidectomy. Histopathologic diagnoses included 42 cPTC, 8 NIFTP, 3 invasive FVPTC, 2 follicular adenomas, and 1 poorly differentiated carcinoma. Excluding 7 indeterminate cases, $89 \%$ (8/9) of nodules classified as NIFTP/FVPTC on FNA demonstrated follicular-patterned lesions on histology (5 NIFTP, 1 invasive FVPTC, 2 follicular adenomas). Cytopathologists prospectively identified cPTC in 95\% (38/40) of cases.

Conclusions: In thyroid FNAs with cytologic features concerning for PTC, NIFTP/FVPTC can be distinguished from cPTC in most cases by assessing a limited number of features. Therefore, it is both feasible and appropriate to attempt to separate NIFTP/FVPTC from cPTC on FNA to promote appropriate clinical management.

\section{Introduction}

$\mathbf{T}$ HE TERM NONINVASIVE FOLLICULAR thyroid neoplasm with papillary-like nuclear features (NIFTP) has recently been proposed to replace noninvasive follicular variant of papillary thyroid carcinoma (FVPTC) (1). The designation of NIFTP rather than carcinoma represents a culmination of work demonstrating that these tumors are a distinct subset of FVPTC with a very indolent clinical course (2-5). The histologic diagnosis of NIFTP relies on a few critical morphologic criteria, including (1) the presence of an entirely follicular architecture with $<1 \%$ papillary structures, (2) the nuclear features of PTC including fine chromatin, pale nuclei, and nuclear grooves, (3) a sharp interface between the tumor and the adjacent thyroid parenchyma, including encapsulated, partially-encapsulated, and well-circumscribed tumors, and (4) the lack of infiltrative growth, capsular invasion, or lymphovascular invasion. Accordingly, NIFTP warrants excision by lobectomy to exclude an invasive FVPTC, classical PTC (cPTC), or other thyroid malignancy, and because NIFTP itself requires excision since it may be a precursor to a more aggressive thyroid tumor. Once the diagnosis of NIFTP is made histologically, however, further therapy such as completion thyroidectomy and/or radioiodine therapy may not be warranted.

Adoption of the NIFTP terminology has significant consequences for thyroid fine-needle aspiration (FNA). The risk of malignancy, assuming NIFTP is classified as nonmalignant, associated with all diagnostic categories of The Bethesda System for Reporting Thyroid Cytopathology (TBSRTC) would decrease. Of particular note, our group demonstrated

\footnotetext{
${ }^{1}$ Department of Pathology and ${ }^{3}$ Department of Medicine, Brigham and Women's Hospital, Harvard Medical School, Boston, Massachusetts.

${ }^{2}$ Department of Pathology, Massachusetts General Hospital, Harvard Medical School, Boston, Massachusetts.

*These authors contributed equally to the work.
} 
that the risk of malignancy of the "suspicious for malignancy" category would decrease by up to $50 \%$ (6), a finding that was supported by Faquin et al. in a multi-institutional study (7). The challenge posed by NIFTP is especially acute with the "suspicious for malignancy" and "malignant" categories, where the FNA diagnosis frequently prompts initial total thyroidectomy, but where more conservative management (i.e., lobectomy) would be preferable if the histologic diagnosis proves to be NIFTP.

Recently, members of our group published the results of a small pilot study retrospectively comparing the cytologic features of NIFTP with those of cPTC (8). The results of this study showed that NIFTP was more likely diagnosed as suspicious for malignancy rather than malignant on cytology and demonstrated a microfollicular predominant architecture. In contrast, the presence of papillae, pseudoinclusions, and psammomatous calcifications were confined to cases of cPTC. Because of the significant implications for patient management, distinction of NIFTP or FVPTC from cPTC is desirable at the time of FNA. The aim of the current study was to determine if cytologists can prospectively distinguish NIFTP from cPTC using the criteria described above.

\section{Materials and Methods}

This clinical investigation was approved by the institutional review board. The study cohort included all thyroid FNAs evaluated from June 1, 2015, to January 15, 2016, at Brigham and Women's Hospital. Thyroid FNAs were performed under ultrasound guidance by staff endocrinologists, without routine on-site evaluation. Aspirates from three to four passes using a 25-gauge needle were collected immediately in CytoLyt (Hologic, Inc., Marlborough, MA), and Papanicolaou-stained ThinPrep slides were prepared using the ThinPrep 2000 (Hologic). All thyroid FNA specimens were diagnosed according to criteria set forth by the Bethesda System for Reporting Thyroid Cytopathology (9) by 1 of 10 staff cytopathologists participating in the study. Study cases were comprised only of "suspicious for malignancy" or "malignant" diagnoses as these are the most likely to prompt total thyroidectomy and therefore present the greatest opportunity to alter clinical management by distinguishing NIFTP/FVPTC from cPTC. For each case, the cytopathologist responsible for rendering the diagnosis as part of routine clinical care also completed a study questionnaire. All questionnaires were completed prospectively, prior to any subsequent surgery. Cases with available surgical follow-up comprised the final study cohort. Resected surgical pathology specimens were reviewed by an endocrine pathology specialist (J.B. and P.S.).

Each questionnaire included entries to record the presence or absence of the following morphologic characteristics: papillae, pseudoinclusions, psammomatous calcifications, microfollicular predominance (or alternatively, a predominance of follicular cells in sheets). If present, the cytopathologist was asked to record the number of pseudoinclusions as frequent $(\geq 3)$ or rare (1-2). Based on these criteria, the cytopathologist was asked to classify the aspirate into 1 of 3 categories: (1) cPTC, based on the presence of papillae, pseudoinclusions, or psammomatous calcifications; (2) NIFTP/ FVPTC, based on microfollicle predominance without papillae, pseudoinclusions, or psammomatous calcifications; or (3) indeterminate, based on the predominance of follicular cells in sheets without papillae, pseudoinclusions, or psammomatous calcifications.

\section{Statistical analysis}

Fisher's exact test was used to evaluate the statistical significance of categorical variables. All $p$-values are two sided, and a level of 0.05 was considered statistically significant. GraphPad Prism v5.02 (GraphPad Software, San Diego, CA) was used to analyze the data.

\section{Results}

\section{Clinical features}

During the study period, 29 thyroid FNA specimens were diagnosed as "suspicious for malignancy" and 69 as "malignant." Surgical follow-up was available for $56(57 \%)$ of these nodules (13 "suspicious for malignancy" and 43 "malignant" on cytology). Liquid-based cytology preparations were available for 53 cases, while smears were the only type of preparations for three cases. Twenty (36\%) were consult cases from outside institutions, and 9 of these contained smear preparations. The 56 nodules were from 52 patients, 38 females and 14 males, with an average age of 47 years (range, 24-87 years). There were 49 initial total thyroidectomies and 3 initial lobectomies. The 3 patients with initial lobectomies had $2 \mathrm{cPTC}$ and 1 FVPTC with capsular invasion; only 1 patient with cPTC underwent completion thyroidectomy. Of the 56 nodules, the final histologic diagnoses were 42 cPTC, 8 NIFTP, 3 FVPTC with invasion, 2 follicular adenomas, and 1 poorly differentiated carcinoma.

\section{Cytologic and histologic features}

Statistical parameters for each of the cytologic features (papillae, pseudoinclusions, frequent pseudoinclusions, psammomatous calcifications, and microfollicular predominance; see Fig. 1) are listed in Table 1, and cytologic and histologic diagnoses, along with the prospective impression, are summarized in Fig. 2. Of the $42 \mathrm{cPTC}, 38(91 \%)$ were favored to be classical at the time of FNA, $3(7 \%)$ were categorized as indeterminate, and 1 case (2\%) was mistaken for NIFTP/FVPTC. Of the 13 follicular-patterned lesions, cytologists categorized 8 (62\%) as NIFTP/FVPTC (5 were NIFTP, 2 were follicular adenomas, and 1 was an FVPTC with invasion), 3 (27\%) as indeterminate, and $2(18 \%)$ as cPTC. Overall, excluding indeterminate cases, if the cytologist favored cPTC, the resection specimen was cPTC in $95 \%$ of cases (38/40), and, if the cytologist favored NIFTP/FVPTC, the resection specimen was a follicular-patterned lesion in $89 \%$ of cases (8/9).

\section{Discordant cases}

Of the 49 cases favored to be either cPTC or NIFTP/FVPTC by cytologists, 3 cases were mistakenly categorized. Two NIFTP/FVPTC were mistakenly categorized as cPTC on cytology. One of these was a NIFTP $(1.1 \mathrm{~cm})$ located adjacent to a cPTC $(1.2 \mathrm{~cm})$, and it is unclear whether the cPTC was inadvertently sampled at the time of FNA. The other was an encapsulated FVPTC with capsular invasion; however, the tumor was largely replaced by FNA site change (Fig. 3), raising the possibility that the nodule previously had a papillary architecture that was obliterated. The third case was a 
A

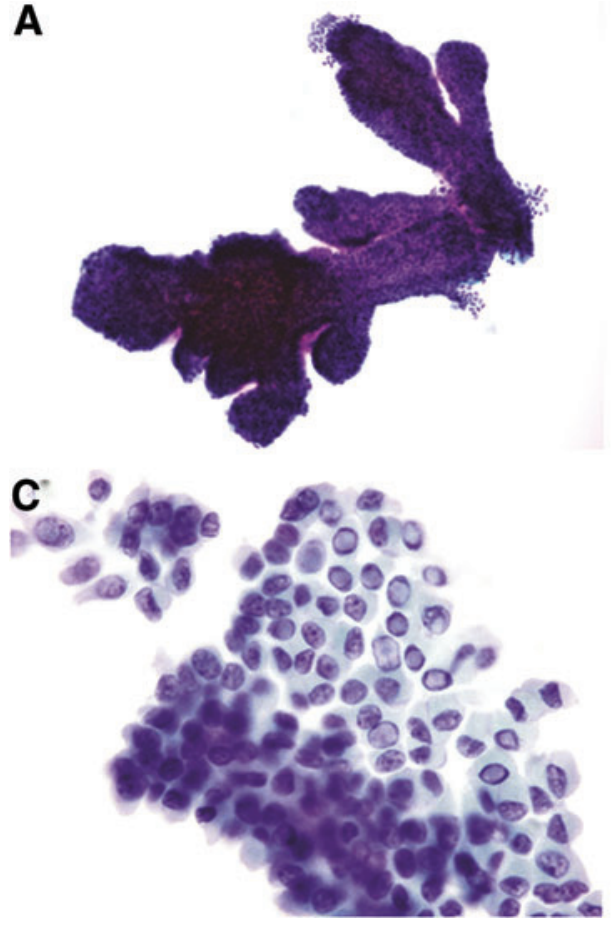

B

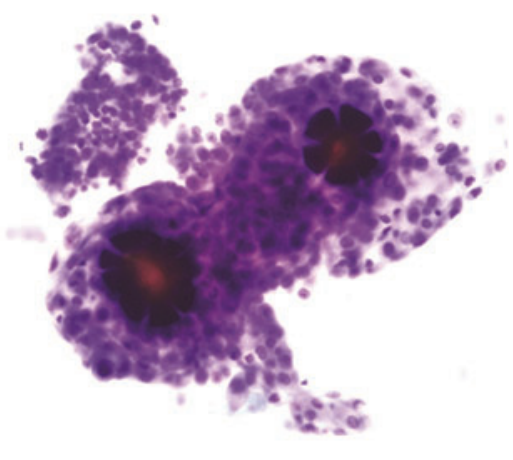

D
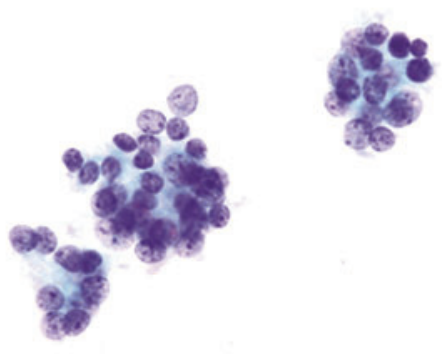

FIG. 1. Cytologic features used to distinguish cPTC from NIFTP/FVPTC. All aspirates were evaluated for evidence of cPTC as shown by the presence of (A) papillary architecture (ThinPrep, Pap stain, $200 \times$ magnification); (B) psammomatous calcifications (ThinPrep [Hologic, Inc.], Pap stain, $400 \times$ magnification); and/or (C) frequent (three or more) nuclear pseudoinclusions (ThinPrep, Pap stain, $1000 \times$ magnification). If these features were absent and there was a predominantly microfollicular architecture (D); ThinPrep, Pap stain, 1000× magnification), then NIFTP/FVPTC was favored. If features of cPTC were absent and sheet-like growth predominated, then the findings were deemed indeterminate. cPTC, classical papillary thyroid carcinoma; NIFTP, noninvasive follicular thyroid neoplasm with papillary-like nuclear features; FVPTC, follicular variant of papillary thyroid carcinoma. Color images available online at www.liebertpub.com/thy

cPTC that was mistakenly categorized as NIFTP/FVPTC (Fig. 4). On cytology, the tumor showed a microfollicularpredominant architecture, with crowded, three-dimensional groups composed of atypical follicular cells with pale chromatin and nuclear grooves. The cytologist identified rare papillary structures, one of which contained a single pseudoinclusion, and favored NIFTP/FVPTC "but with a low degree of confidence." On histology, although the tumor had papillae (and was therefore characterized as cPTC), much of the tumor had a follicular architecture.

\section{Discussion}

The recently introduced terminology of NIFTP (1) represents a paradigm shift in the diagnosis of PTC, recognizing that tumors previously classified as encapsulated, partially encapsulated or well-circumscribed FVPTC without capsular penetration or lymphovascular invasion have little, if any, metastatic potential or risk of recurrence $(4,5,10-13)$. Furthermore, NIFTP tend to harbor $R A S$ and $P A X 8 / P P A R \gamma$ mutations and lack the $B R A F^{V 600 E}$ mutation that is associated

Table 1. Cytomorphologic Features and Associated Histologic Diagnosis

\begin{tabular}{|c|c|c|c|c|c|}
\hline Histologic diagnosis ${ }^{\mathrm{a}}$ & Total & Papillae $^{\mathrm{b}}$ & $\begin{array}{c}\text { Nuclear } \\
\text { pseudoinclusions }^{\mathrm{c}} \\
\text { [if } \geq 3]\end{array}$ & $\begin{array}{l}\text { Psammomatous } \\
\text { calcifications }{ }^{\mathrm{d}}\end{array}$ & $\begin{array}{l}\text { Microfollicular } \\
\text { predominant }^{\mathrm{e}}\end{array}$ \\
\hline cPTC & 42 & 30 & $35[22]$ & 7 & 2 \\
\hline NIFTP/FVPTC/Adenoma & 13 & 2 & $2[0]$ & 0 & 8 \\
\hline FVTPC & 3 & 1 & $1[0]$ & 0 & 1 \\
\hline NIFTP & 8 & 1 & $1[0]$ & 0 & 5 \\
\hline Adenoma & 2 & 0 & $0[0]$ & 0 & 2 \\
\hline
\end{tabular}

${ }^{\mathrm{a}}$ For the purposes of this table, results for the poorly differentiated carcinoma are excluded.

${ }^{\mathrm{b}} p=0.0007$.

$p<0.0001$

${ }^{\mathrm{d}} p=0.179$.

e $p<0.0001$.

cPTC, classical papillary thyroid carcinoma; NIFTP, noninvasive follicular thyroid neoplasm with papillary-like nuclear features; FVPTC, follicular variant of papillary thyroid carcinoma. 


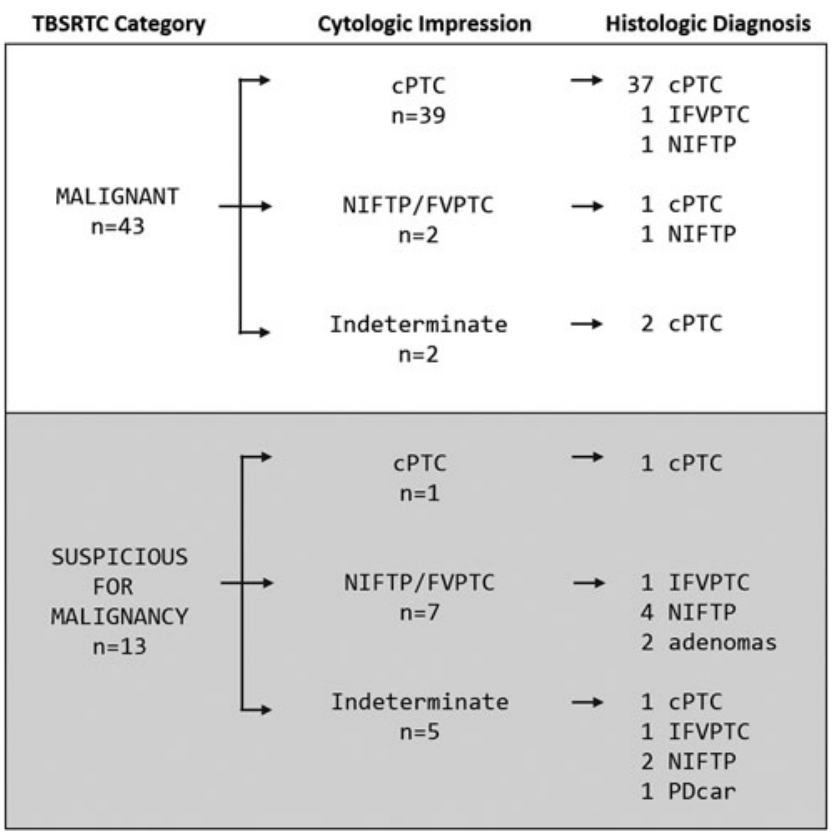

FIG. 2. Histologic diagnoses compared to cytologic impression. TBSRTC, The Bethesda System for Reporting Thyroid Cytopathology; IFVPTC, follicular variant of papillary thyroid carcinoma with invasion/infiltration; PDcar, poorly differentiated carcinoma.

with high-risk histopathologic features (14-16). Therefore, the behavior and molecular alterations of NIFTP indicate that these tumors are more similar to follicular adenomas and follicular carcinomas than they are to cPTC. As is true with follicular neoplasms, diagnostic excision (typically by lobectomy) is needed to establish the diagnosis of NIFTP and to exclude alternative diagnoses particularly encapsulated FVPTC with invasion, infiltrative FVPTC, and cPTC. Moreover, NIFTP requires surgical management since it may be a precursor to a more aggressive thyroid tumor. Once the diagnosis of NIFTP is made histologically, however, further therapy such as completion thyroidectomy and/or radioiodine therapy may not be warranted.

The introduction of NIFTP poses significant challenges for cytopathologists in classifying thyroid nodules on FNA. Tumors historically classified as FVPTC do not typically exhibit the full range of cytologic features associated with cPTC and as a result have tended to be classified in the indeterminate categories of the TBSRTC framework, with "malignant" aspirates preferentially representing cPTC (17). Architectural alterations of NIFTP/FVPTC also overlap significantly with aspirates in the "suspicious for a follicular neoplasm" category (18). Although NIFTP cases are distributed among all TBSRTC categories $(6,7)$, in current practice NIFTP is frequently associated with the "suspicious for malignancy" diagnosis (6). Consequently, many patients with NIFTP are triaged to total thyroidectomy when lobectomy alone would have been the adequate therapy.

In order to triage patients with NIFTP to more conservative clinical management, cytopathologists need to be able to distinguish NIFTP/FVPTC from cPTC. To determine whether this distinction can be made in a reliable manner, we prospectively classified aspirates originally diagnosed as "suspicious for malignancy" or "malignant" using criteria we had previously identified as helpful in distinguishing between NIFTP/FVPTC and cPTC (8). These criteria were sufficient to permit classification as either NIFTP/FVPTC or cPTC in $87.5 \%$ (49/56) of cases with accurate distinction between cPTC and other follicular patterned lesions (including NIFTP, FVPTC, and follicular adenoma) in 94\% (46/ 49) of classified cases. Thus, in cases where nuclear features already raise significant concern for the diagnosis of PTC, cytologists are generally successful at separating NIFTP/ FVPTC from cPTC by looking for a predominantly microfollicular architectural pattern in NIFTP/FVPTC and frequent nuclear pseudoinclusions, papillae, and/or psammomatous calcifications in cPTC.

In practical terms, if the pathologist's cytologic impression had been used to limit extent of surgery for our study population, management would have been altered for 8 patients. These 8 patients favored to have NIFTP/FVPTC on FNA would have had initial lobectomy instead of total thyroidectomy; only 1 of these 8 patients would have been subsequently triaged to completion thyroidectomy after an initial diagnostic lobectomy demonstrated focal features warranting a diagnosis of cPTC. Altered management would have the greatest impact in the "suspicious for malignancy" category, where it would have affected 54\% (7/13) of cases, while only altering management in $2 \%(1 / 43)$ of "malignant" cases. This difference reflects the fact that $\mathrm{CPTC}$ diagnoses are preferentially represented in the "malignant" category (93\%; 40/43 nodules), while the "suspicious for malignancy" category is mostly represented by follicular-patterned lesions (only $15 \%$ cPTC; $2 / 13$ nodules). In our cohort there were very few cPTC with a preceding "suspicious for malignancy" diagnosis on FNA. This may suggest that triaging all patients with a "suspicious for malignancy" diagnosis to lobectomy instead of total thyroidectomy, would be sufficient. However, in previous cohorts studied by our group $(6,17)$ and others (7), the "suspicious for malignancy" category included a higher percentage of cPTCs, indicating further refinement of the "suspicious for malignancy" category could be helpful in guiding initial surgical strategy.

If, as our study suggests, NIFTP/FVPTC can be prospectively recognized, the appropriate classification of such lesions should be addressed in a future revision of the TBSRTC. In many ways, the limitations in recognizing NIFTP by FNA parallel similar constraints in classifying follicular neoplasms. As is true for follicular neoplasms, cytology alone cannot render a definitive diagnosis of NIFTP. Histologic examination is needed to exclude alternative diagnoses where the tumor is infiltrative (infiltrative FVPTC), is encapsulated but with capsular or vascular invasion (encapsulated FVPTC with invasion), has focal papillary architecture (cPTC), has high mitotic activity, necrosis or solid growth (poorly differentiated carcinoma), or lacks sufficient nuclear features to warrant the diagnosis of NIFTP (follicular adenoma or follicular carcinoma). Nevertheless, in most instances where NIFTP is suspected cytologically, the histologic diagnosis will not be a malignancy. Therefore, we believe there are two possible approaches currently: (1) suspected NIFTP lesions could be classified in the "suspicious for a follicular neoplasm" category; or (2) suspected NIFTP lesions could be classified as "suspicious for malignancy" but with a comment that NIFTP/FVPTC is likely/ favored. With appropriate education, this approach would achieve triage similar to usual aspirates in the "suspicious for a follicular neoplasm" category, which are typically referred for 


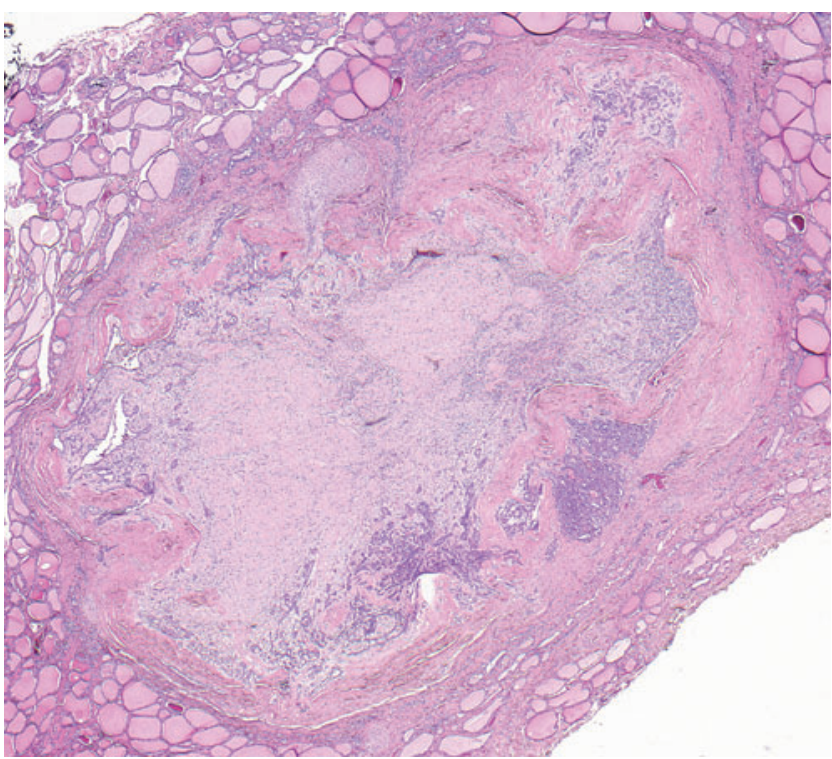

FIG. 3. FVPTC with capsular invasion. There is extensive fibrosis consistent with fine needle aspiration (FNA) site change. The corresponding FNA was favored to be cPTC and papillae were noted, raising the possibility that the tumor had a papillary architecture which was obliterated by the FNA (H\&E; $100 \times$ magnification). Color images available online at www.liebertpub.com/thy

a diagnostic lobectomy, possibly preceded by molecular testing if desired. The majority of patients with a suspected NIFTP will require no further surgery, with completion lobectomy reserved for individuals with a malignant histologic diagnosis. Suspected NIFTP/FVPTC may ultimately merit a separate category in a future iteration of the TBSRTC, but only if reproducibly recognizable criteria can be identified that will allow reliable distinction from other diagnostic categories. Additionally, although NIFTP/FVPTC and cPTC already tend to stratify into the "suspicious for malignancy" and "malignant" TBSRTC categories, it is important to identify potential criteria to distinguish "suspicious for malignancy" and "malignant" categories to preserve the historically nearly $100 \%$ positive predictive value of a "malignant" cytologic diagnosis.

There are limitations of our study that warrant some caution. Our study was conducted over a relatively short time interval with a limited number of cases from a single academic center. The criteria that we focused on studying were recognized and applied largely in liquid based cytology (ThinPrep) preparations. It is possible that these criteria may not be applicable to the same degree with smears or that other criteria may prove more useful in distinguishing NIFTP/ FVPTC from cPTC with other preparation methods. Our study also represents an intentionally simplified approach to making the distinction between NIFTP/FVPTC and cPTC, as it was our hope that these criteria would be easily adopted in routine clinical practice. It is possible that additional cytologic criteria or correlation with ultrasonographic findings (19) may enable even better distinction between these entities. Certainly, verification in other laboratories including practitioners in nonacademic settings will help establish optimal criteria that can be used in routine practice settings.

In conclusion, NIFTP is an indolent tumor that has historically been classified on cytology along with variants of PTC. Our study indicates that simple cytologic and architectural features (i.e., papillae, pseudoinclusions, psammomatous calcifications, and microfollicular architecture) can be used to distinguish prospectively the majority of NIFTP and other follicular-patterned lesions from cPTC. Therefore, cytopathologists should be able to diagnose and appropriately triage the majority of patients with putative NIFTP/FVPTC for consideration of diagnostic lobectomy while also recognizing cPTC and triaging those for total thyroidectomy.
FIG. 4. A cPTC favored to be NIFTP/FVPTC on cytology. This case of a follicular-predominant cPTC illustrates a potential pitfall of diagnosing NIFTP/FVPTC on cytology. (A) The tumor displayed a microfollicular-predominant architecture (ThinPrep, Pap stain, $200 \times$ magnification). (B) Follicles were composed of crowded follicular cells with pale chromatin and nuclear grooves (ThinPrep, Pap stain, $1000 \times$ magnification). (C) There were rare papillary-like structures, one containing a single pseudoinclusion (arrow) (ThinPrep, Pap stain, $1000 \times$ magnification). (D) Histologic sections revealed a tumor that was predominantly follicular-patterned (inset, $600 \times$ magnification) but contained a small focus of papillary architecture, warranting a diagnosis of cPTC (H\&E, $200 \times$ magnification). Color images available online at www.liebertpub.com/thy
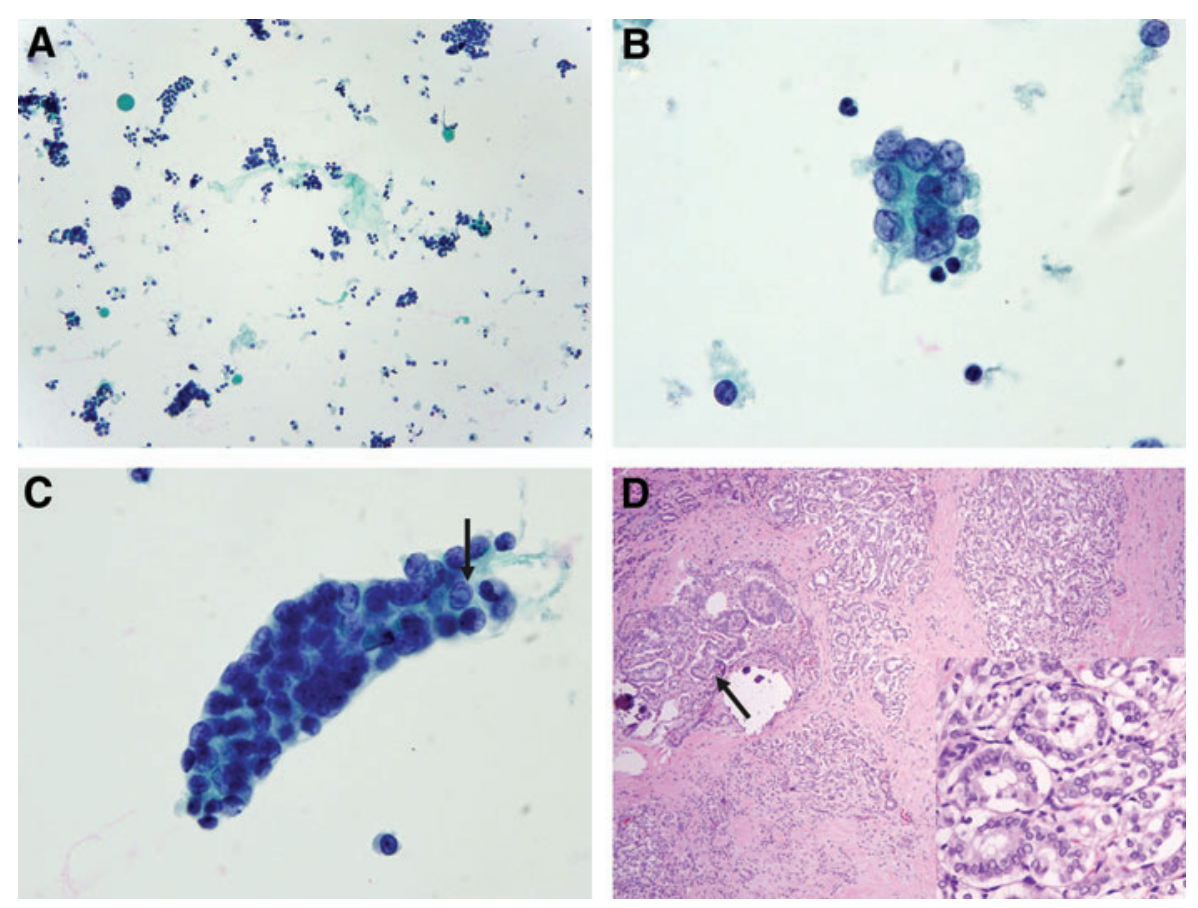


\section{Author Disclosure Statement}

Erik K. Alexander has acted as a paid member of the Scientific Advisory Board for Asuragen Inc. and as a paid consultant for Veracyte Inc. In addition, his institution has received research support from both Asuragen and Veracyte. No competing financial interests exist for the other authors.

\section{References}

1. Nikiforov YE, Seethala RR, Tallini G, Baloch ZW, Basolo F, Thompson LDR, Barletta JA, Wenig BM, Al Ghuzlan A, Kakudo K, Giordano TJ, Alves VA, Khanafshar E, Asa SL, El-Naggar AK, Gooding WE, Hodak SP, Lloyd R V, Maytal G, Mete O, Nikiforova MN, Nosé V, Papotti M, Poller DN, Sadow PM, Tischler AS, Tuttle RM, Wall KB, LiVolsi VA, Randolph GW, Ghossein RA 2016 Nomenclature Revision for Encapsulated Follicular Variant of Papillary Thyroid Carcinoma. JAMA Oncol 2:1023-1029.

2. Rivera M, Ricarte-Filho J, Knauf J, Shaha A, Tuttle M, Fagin JA, Ghossein RA 2010 Molecular genotyping of papillary thyroid carcinoma follicular variant according to its histological subtypes (encapsulated vs infiltrative) reveals distinct BRAF and RAS mutation patterns. Mod Pathol 23:1191-1200.

3. Ganly I, Wang L, Tuttle RM, Katabi N, Ceballos GA, Harach HR, Ghossein R 2015 Invasion rather than nuclear features correlates with outcome in encapsulated follicular tumors: further evidence for the reclassification of the encapsulated papillary thyroid carcinoma follicular variant. Hum Pathol 46:657-664.

4. Howitt BE, Jia Y, Sholl LM, Barletta JA 2013 Molecular alterations in partially-encapsulated or well-circumscribed follicular variant of papillary thyroid carcinoma. Thyroid 23:1256-1262.

5. Vivero M, Kraft S, Barletta JA 2013 Risk stratification of follicular variant of papillary thyroid carcinoma. Thyroid 23:273-279.

6. Strickland KC, Howitt BE, Marqusee E, Alexander EK, Cibas ES, Krane JF, Barletta JA 2015 The impact of noninvasive follicular variant of papillary thyroid carcinoma on rates of malignancy for fine-needle aspiration diagnostic categories. Thyroid. 25:987-992.

7. Faquin WC, Wong LQ, Afrogheh AH, Ali SZ, Bishop JA, Bongiovanni M, Pusztaszeri MP, VandenBussche CJ, Gourmaud J, Vaickus LJ, Baloch ZW 2016 Impact of reclassifying noninvasive follicular variant of papillary thyroid carcinoma on the risk of malignancy in The Bethesda System for Reporting Thyroid Cytopathology. Cancer Cytopathol 124:181-187.

8. Howitt BE, Chang S, Eszlinger M, Paschke R, Drage MG, Krane JF, Barletta JA 2015 Fine-needle aspiration diagnoses of noninvasive follicular variant of papillary thyroid carcinoma. Am J Clin Pathol 144:850-857.

9. Cibas ES, Ali SZ 2009 The Bethesda System for Reporting Thyroid Cytopathology. Thyroid 19:1159-1165.

10. Rivera M, Ricarte-Filho J, Knauf J, Shaha A, Tuttle M, Fagin JA, Ghossein RA 2010 Molecular genotyping of papillary thyroid carcinoma follicular variant according to its histological subtypes (encapsulated vs infiltrative) reveals distinct BRAF and RAS mutation patterns. Mod Pathol 23:1191-1200.

11. Liu J, Singh B, Tallini G, Carlson DL, Katabi N, Shaha A, Tuttle RM, Ghossein RA 2006 Follicular variant of papillary thyroid carcinoma: a clinicopathologic study of a problematic entity. Cancer 107:1255-1264.

12. Piana S, Frasoldati A, Di Felice E, Gardini G, Tallini G, Rosai J 2010 Encapsulated well-differentiated follicularpatterned thyroid carcinomas do not play a significant role in the fatality rates from thyroid carcinoma. Am J Surg Pathol 34:868-872.

13. Thompson LD 2016 Ninety-four cases of encapsulated follicular variant of papillary thyroid carcinoma: A name change to Noninvasive Follicular Thyroid Neoplasm with Papillary-like Nuclear Features would help prevent overtreatment. Mod Pathol 29:698-707.

14. Lim JY, Hong SW, Lee YS, Kim B-W, Park CS, Chang HS, Cho JY 2013 Clinicopathologic implications of the BRAF(V600E) mutation in papillary thyroid cancer: a subgroup analysis of 3130 cases in a single center. Thyroid 23:1423-1430.

15. Xing M, Alzahrani AS, Carson KA, Shong YK, Kim TY, Viola D, Elisei R, Bendlová B, Yip L, Mian C, Vianello F, Tuttle RM, Robenshtok E, Fagin JA, Puxeddu E, Fugazzola L, Czarniecka A, Jarzab B, O’Neill CJ, Sywak MS, Lam AK, Riesco-Eizaguirre G, Santisteban P, Nakayama H, Clifton-Bligh R, Tallini G, Holt EH, Sýkorová V 2015 Association between BRAF V600E mutation and recurrence of papillary thyroid cancer. J Clin Oncol 33:42-50.

16. Howitt BE, Paulson VA, Barletta JA 2015 Absence of BRAF $\mathrm{V} 600 \mathrm{E}$ in non-infiltrative, non-invasive follicular variant of papillary thyroid carcinoma. Histopathology 67:579-582.

17. VanderLaan PA, Marqusee E, Krane JF 2012 Features associated with locoregional spread of papillary carcinoma correlate with diagnostic category in the Bethesda System for reporting thyroid cytopathology. Cancer Cytopathol 120:245-253.

18. Ustun B, Chhieng D, Prasad ML, Holt E, Hammers L, Carling T, Udelsman R, Adeniran AJ 2014 Follicular variant of papillary thyroid carcinoma: accuracy of FNA diagnosis and implications for patient management. Endocr Pathol 25:257-264.

19. Rhee SJ, Hahn SY, Ko ES, Ryu JW, Ko EY, Shin JH 2014 Follicular variant of papillary thyroid carcinoma: distinct biologic behavior based on ultrasonographic features. Thyroid 24:683-688.

Address correspondence to:

Jeffrey F. Krane, MD, PhD

Department of Pathology Brigham and Women's Hospital

75 Francis Street

Boston, MA 02115

E-mail: jkrane@partners.org 